\title{
Assessing the validity of RMR equations in healthy Danish community-dwelling older adults
}

\section{Abstract}

Background: Estimating basal energy requirements is important in terms of assessing energy balance and targeting nutritional support. Assessing Resting Metabolic Rate (RMR) via indirect calorimetry is considered a reliable way of estimating basal energy requirements. Several RMR equations have been developed during the past century through indirect calorimetry, but only few have specifically focused on older adults (>65 yrs). This may lessen the validity of the equations in relation to older adults, as body composition which is an important determinant for energy requirement changes considerably with age. Additional studies are therefore needed to assess the validity of available RMR equations in specific populations of older adults.

Aim: The aim of the present study was to investigate the validity of commonly available RMR equations in a group of older apparently healthy community-dwelling Danes $(65+\mathrm{y})$.

Methods: RMR was measured by indirect calorimetry $\left.{ }_{\mathrm{m}} \mathrm{RMR}\right)$ in 42 older individuals (10 females and 32 males; 65-81 years) participating in the CALM intervention study. Oxygen consumption and carbon dioxide production was measured for 25 min using a ventilated open hood system (Oxycon Pro 4; Jeager, Hoechberg, Germany). Predicted RMR ( ${ }_{\mathrm{p}} \mathrm{RMR}$ ) was calculated using four different equations; the Harris-Benedict (1919) (HB), the Mifflin et al. (1990), the Lührmann et al. (2002) and the Henry et al. (2005) equations. The ratio between ${ }_{m} R M R$ and ${ }_{p} R M R\left(R M R_{\text {ratio }}\right.$ ) as well as the predictive ability (percentage of individual ${ }_{p} R M R$ within $+/-10 \%$ of $\left.{ }_{m} R M R\right)$ were calculated as a measure of validity.

Results: $\mathrm{RMR}_{\text {ratio }}$ for both sexes combined was $0.95 \pm 0.08$ for HB, $0.97 \pm 0.07$ for Mifflin et al., $0.92 \pm 0.06$ for Lürhmann et al. and $0.94 \pm 0.07$ for the Henry equations, respectively. The predictive ability was $73.8 \%(78.1 \%$ males and $60.0 \%$ of females, $p=0.410)$ for the HB, $81.0 \%(81.3 \%$ of males, and $80.0 \%$ of females, $\mathrm{p} \approx 1.00)$ for the Mifflin et al., $54.8 \%(56.3 \%$ of males and $50.0 \%$ of females, $\mathrm{p} \approx 1.00$ ) for the Lührmann et al. equation, and $59.5 \%$ (59.4\% of males and $60.0 \%$ of females, $\mathrm{p} \approx 1.00)$ for the Henry equations, respectively.

Conclusion: In the present study, we found that the Mifflin equation was the most valid equations for predicting the RMR of older apparently healthy community-dwelling Danes. Generally, RMR $\mathrm{R}_{\text {ratios }}$ and predictive ability were slightly lower for the H-B and Henry equations and lowest for the Lürhmann equation.

\section{Conflict of Interest}

There is no conflict of interest 